\title{
Correction to: Comparison of Intraoperative Frozen Section with Preoperative CA125, T.Inhibin and Adnex IOTA Model in the Surgical Care of Women with Ovarian Tumors in a Tertiary Care Hospital
}

\author{
Ritu Mehta ${ }^{1} \cdot$ Rashmi Yadav ${ }^{1}$
}

Published online: 2 June 2020

(c) Association of Gynecologic Oncologists of India 2020

Correction to: Indian Journal of Gynecologic Oncology (2020) 18:52 https://doi.org/10.1007/s40944-020-00397-5

In the original article publication, the term "Adenexa" is incorrectly published in the title and several places in the article. The correct term is "Adnex".

The original article has been corrected.

Publisher's Note Springer Nature remains neutral with regard to jurisdictional claims in published maps and institutional affiliations.

The original article can be found online at https:// doi.org/10.1007/s40944-020-00397-5.

Rashmi Yadav

drrashmidv@gmail.com

Ritu Mehta

doctorritumehta@gmail.com

1 Indian Naval Hospital Ship Asvini, Mumbai, India 